\title{
Near real-time air quality forecasts using the NASA GEOS model
}

\section{K. Emma Knowland}

USRA/GESTAR

NASA Global Modeling and Assimilation Office (GMAO)

In collaboration with:

GMAO: Christoph Keller, Lesley Ott, Steven Pawson, Emily Saunders, Pamela Wales Atmospheric Chemistry and Dynamics Lab: Bryan Duncan, Melanie Follette-Cook, Junhua Liu, Julie Nicely 


\section{NASA GMAO global meteorology and chemistry products}

\section{GEOS}
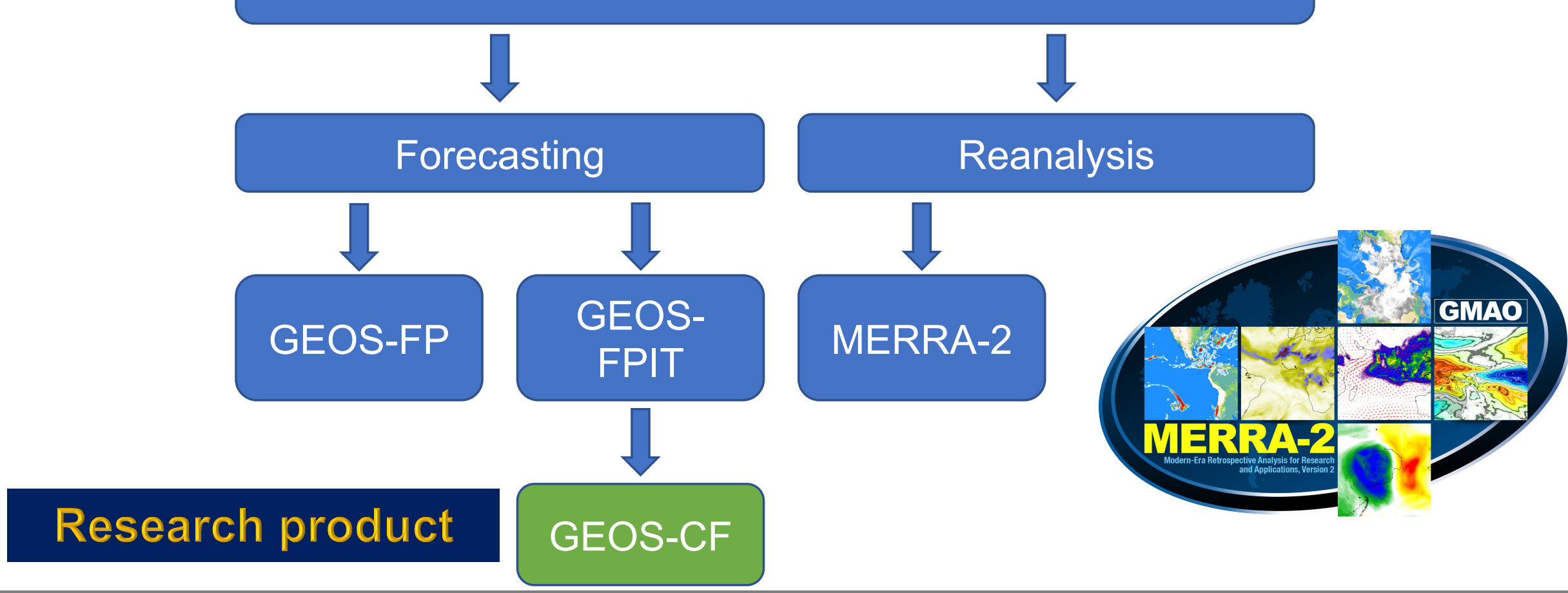

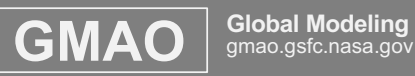




\section{NASA's composition forecast (GEOS-CF)}

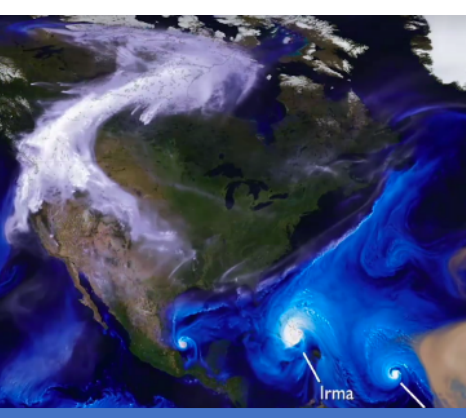

\section{GEOS FPIT}

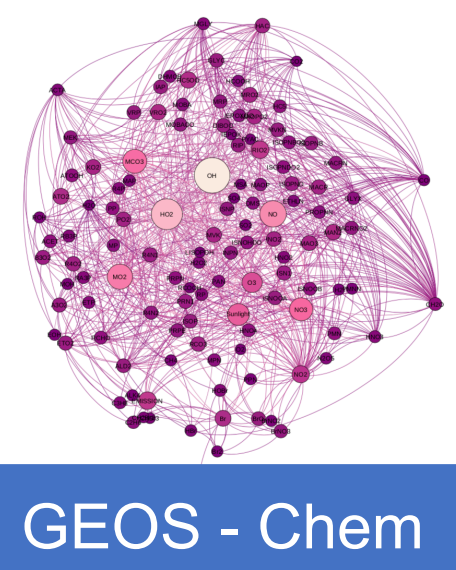

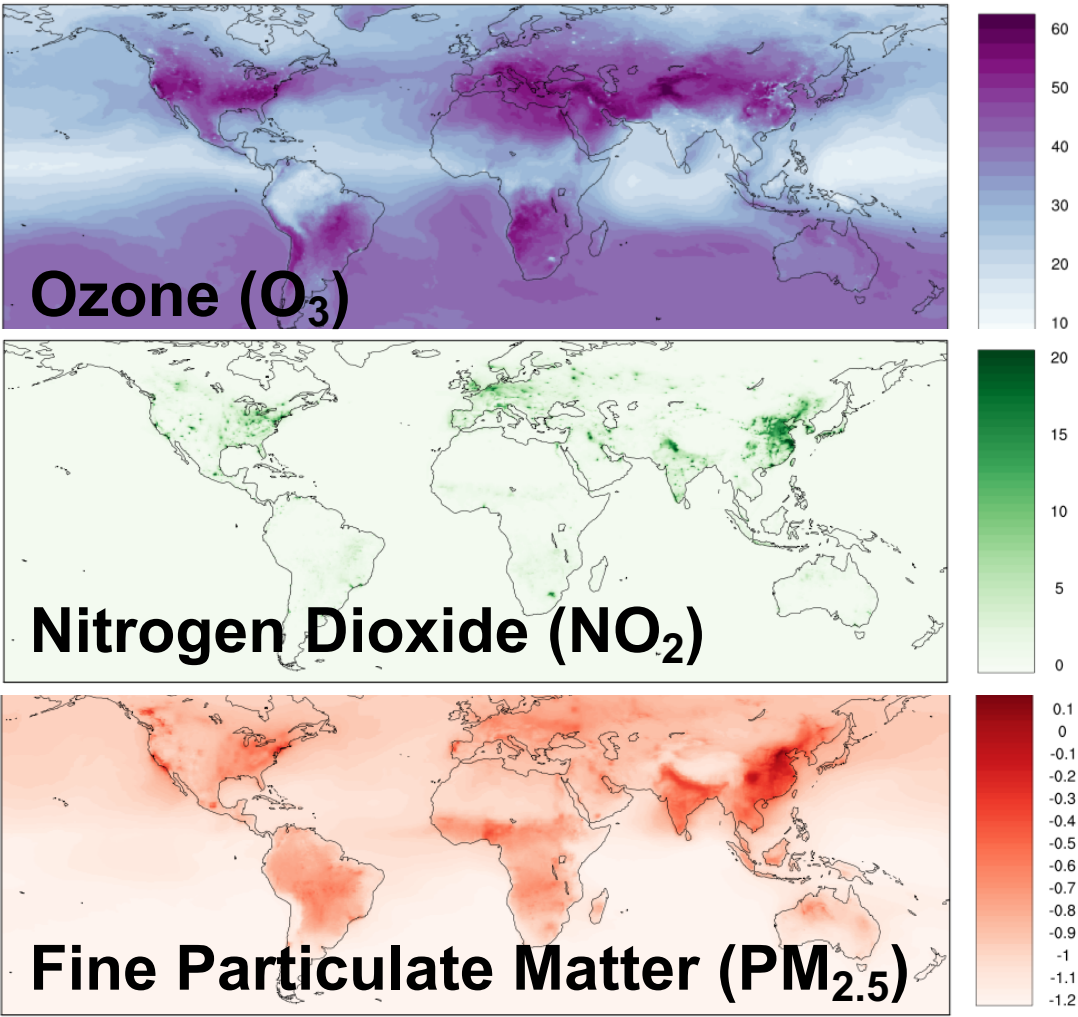

k.e.knowland@nasa.gov christoph.a.keller@nasa.gov

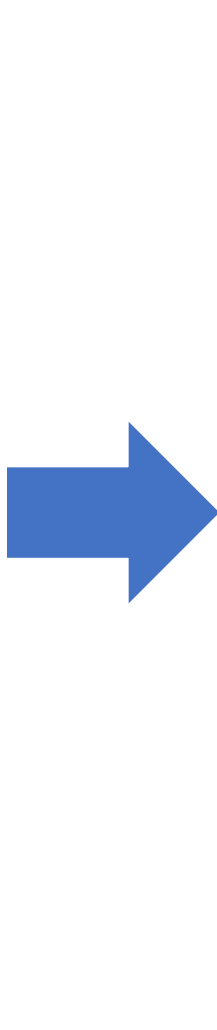

Fine Particulate Matter ( $\left.\mathbf{P M}_{2.5}\right)$ 


\section{NASA GMAO's Composition Forecast}

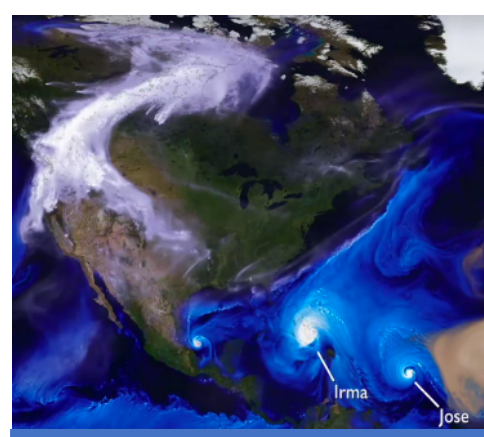

GEOS NWP
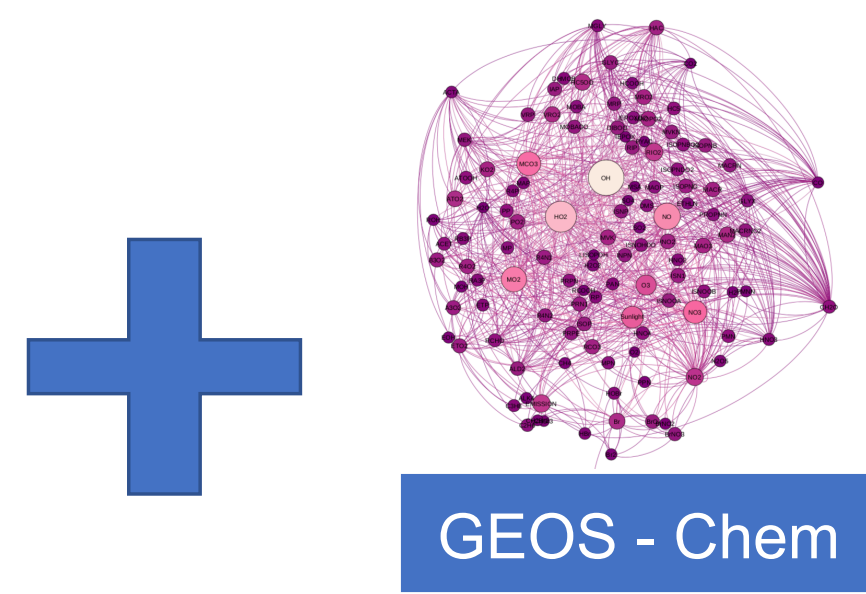

\section{Research product}




\section{GEOS-Chem is a state-of-the science chemistry transport model}

Tropospheric and Stratospheric full chemistry

$>250$ reactive species, 725 reactions

$>100+$ user/developer groups worldwide

$>$ Updated version is released about every year

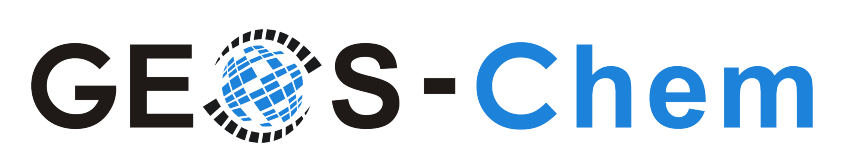




\section{Daily composition forecast}

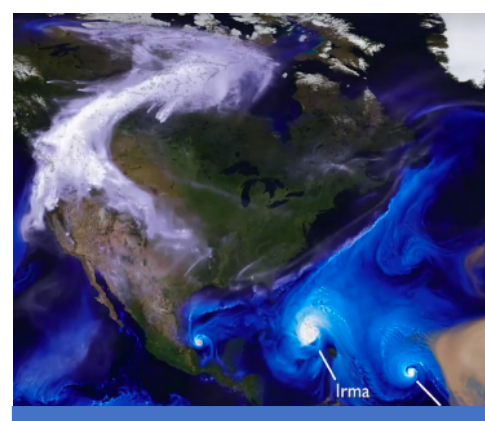

\section{GEOS - CF}

\section{One 5-day forecast per day}

GEOS NWP

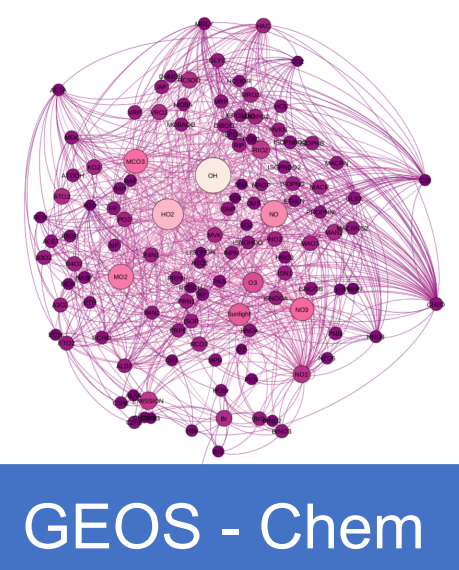




\section{Daily composition forecast}

\section{GEOS - CF}

\section{One 5-day forecast per day} $>1$-day hindcast "analysis" $>5$-day forecast

\section{GEOS NWP}

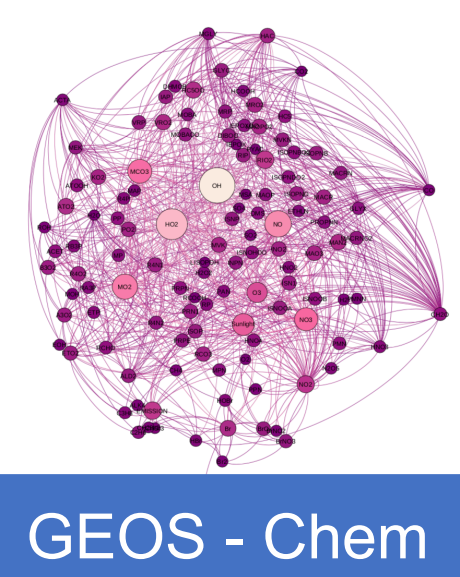




\section{Daily composition forecast}

\section{GEOS - CF}

One 5-day forecast per day

GEOS NWP

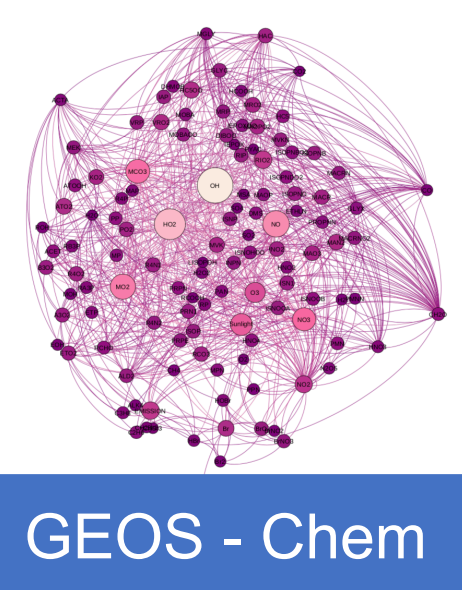

$>5$-day forecast

$>c 360\left(0.25^{\circ}, \sim 25 \times 25 \mathbf{k m}^{2}\right)$ resolution, 72 model layers 


\section{Daily composition forecast}

\section{GEOS - CF}

One 5-day forecast per day

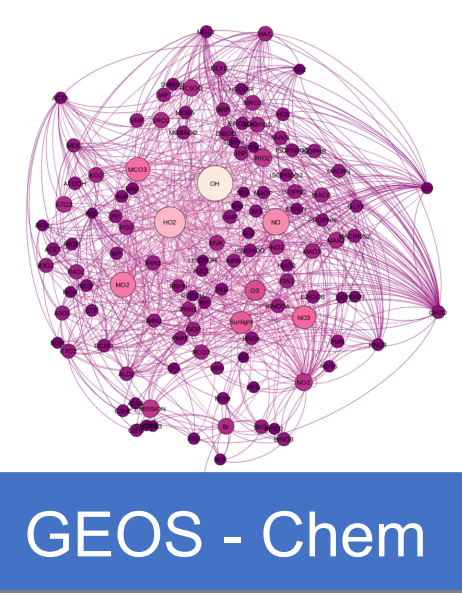

$>$ 5-day forecast

$>c 360\left(0.25^{\circ}, \sim 25 \times 25 \mathbf{k m}^{2}\right)$ resolution, 72 model layers $>\mathrm{O}_{3}, \mathrm{NO}_{\mathrm{x}}, \mathrm{VOCs}, \mathrm{PM} \ldots$
GEOS NWP
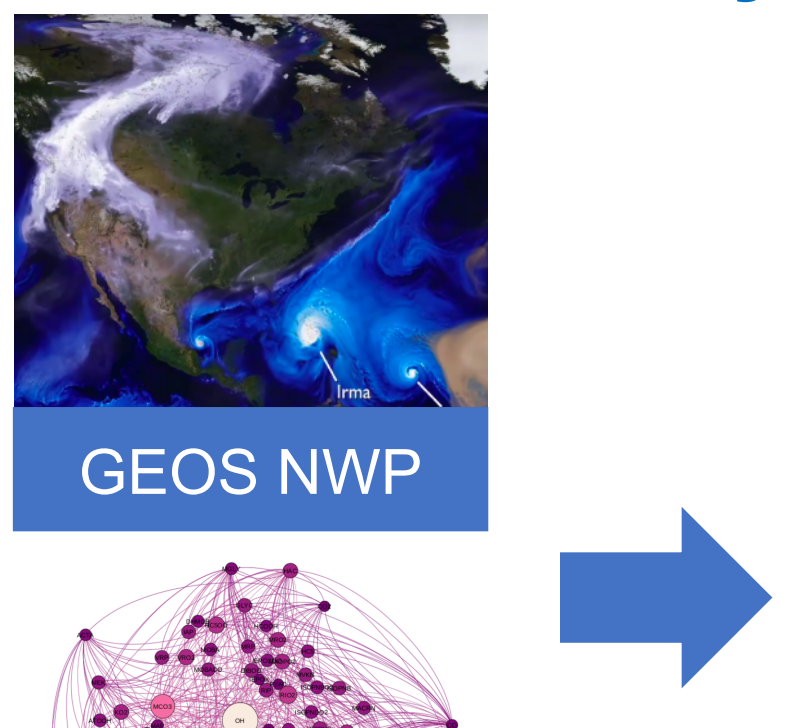


\section{Daily composition forecast}

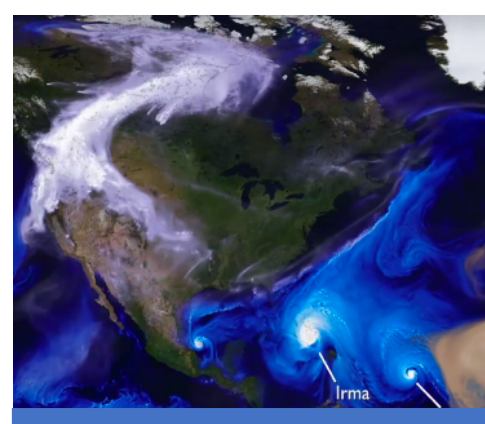

GEOS NWP

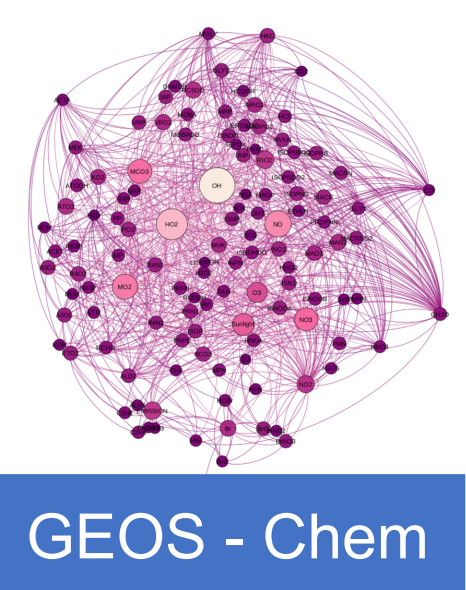

$>1$-day hindcast

$>$ 5-day forecast

$>c 360\left(0.25^{\circ}, \sim 25 \times 25 \mathrm{~km}^{2}\right)$

$>15$ minute "surface"

$>1$-hour average and instantaneous 2D \& 3D 


\section{Daily composition forecast}

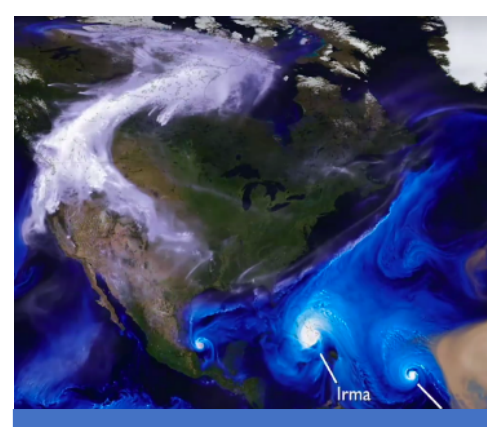

GEOS NWP

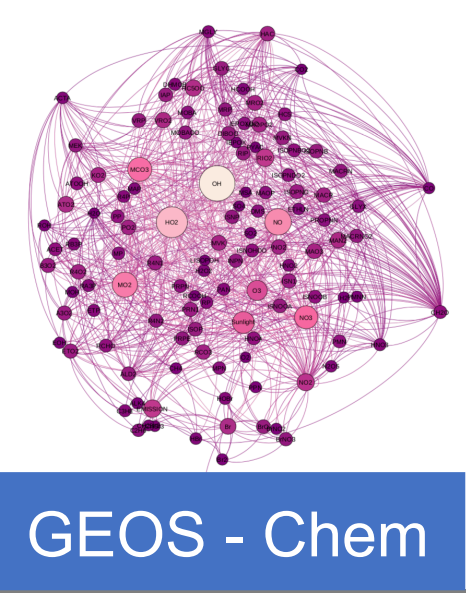

\section{GEOS - CF}

One 5-day forecast per day

$>1$-day hindcast

$>5$-day forecast

$>c 360\left(0.25^{\circ}, \sim 25 \times 25 \mathrm{~km}^{2}\right)$

$>1$ January 2018 - NRT 


\section{Chemistry is not cheap!}

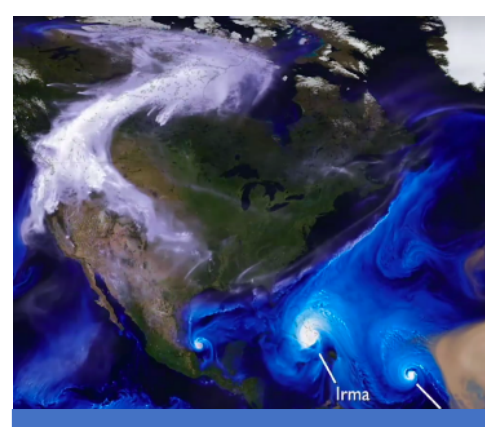

GEOS NWP

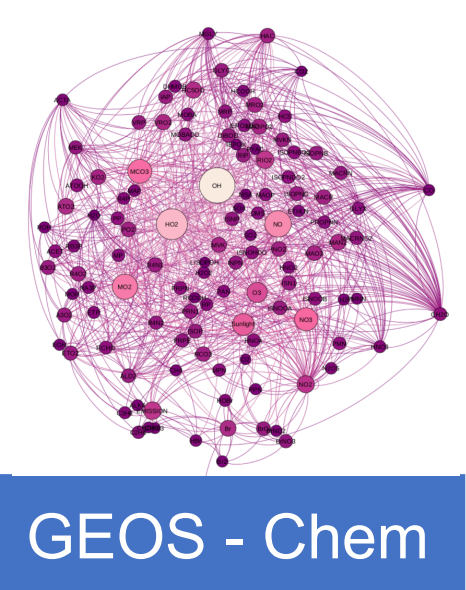

\section{GEOS - CF}

Run on NASA's Center for

Climate Simulation supercomputer

$>$ using the computing power equivalent to 3500 personal computers. 


\section{High-Resolution Global Simulation}

\section{GEOS - CF}

2017-10-01 00:30 UTC

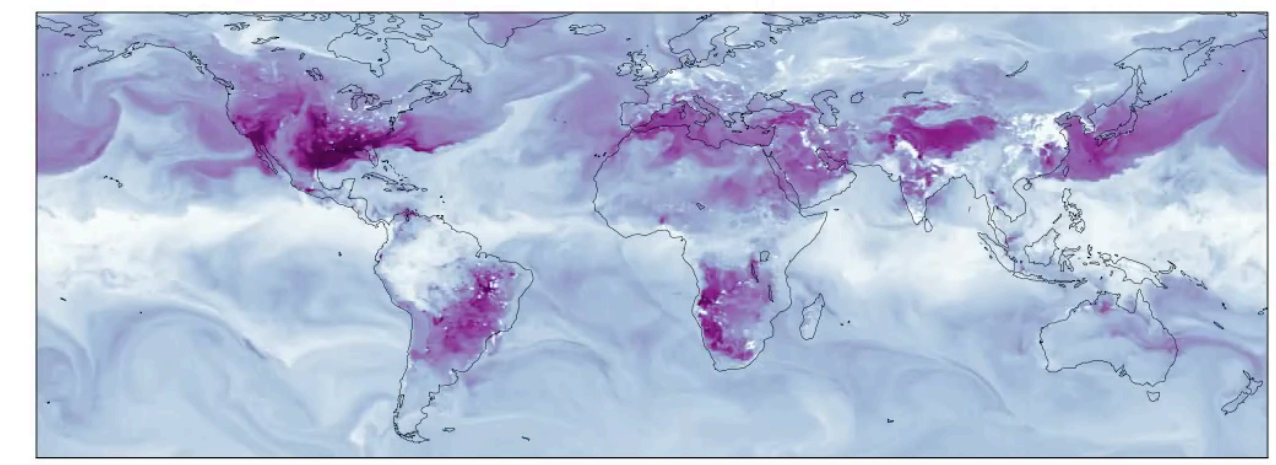

10

20

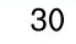

Surface ozone [ppbv]
$25 \mathrm{~km} \times 25 \mathrm{~km}$ (16 miles)

$>$ Highest horizontal resolution of a global atmospheric composition forecast

$>10 \times$ higher than conventional global atmospheric chemistry simulations. 


\section{Case study: agricultural fires in India}

Delhi, India, 2017-10-26 00:00 UTC
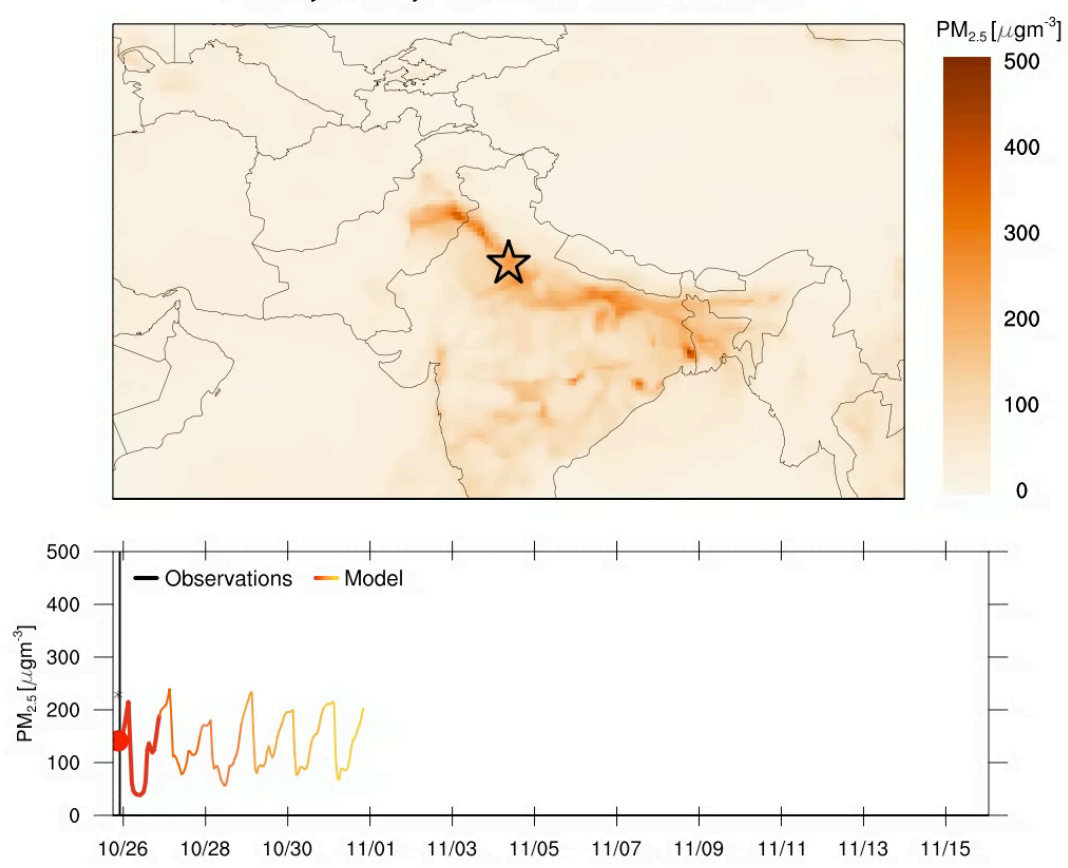

Observation

$0+1+2+3+4+5$

GEOS-CF
MODIS fires Nov 01, 2017

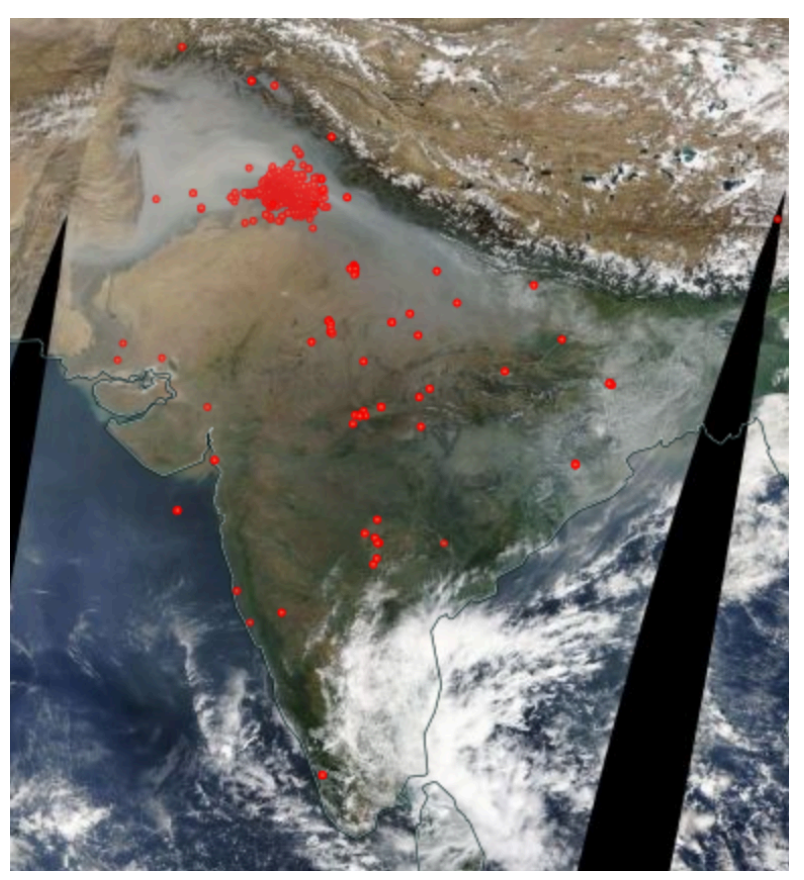




\section{Daily composition forecast}

\section{GEOS - CF}

$>$ Annual emission scale factors based on satellite $>$ Scale factors applied to emissions for diurnal and weekly variations

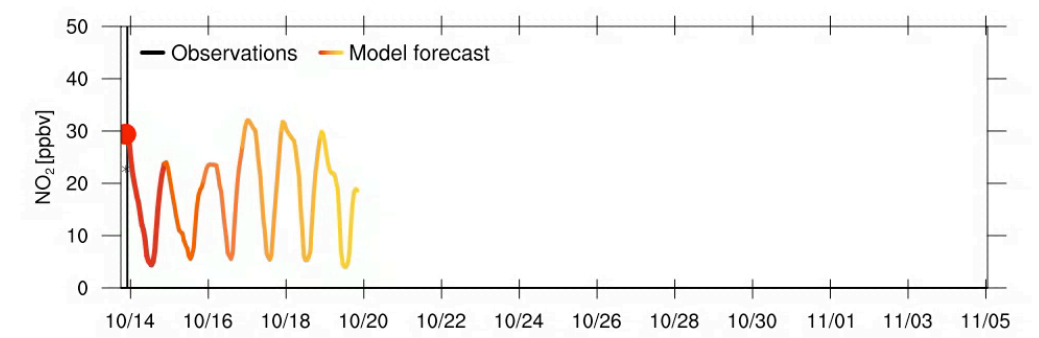

Observation

$0+1+2+3+4+5 \quad$ GEOS-CF 
Where to find GEOS-CF

\section{Output available at fluid.nccs.nasa.gov/cf}

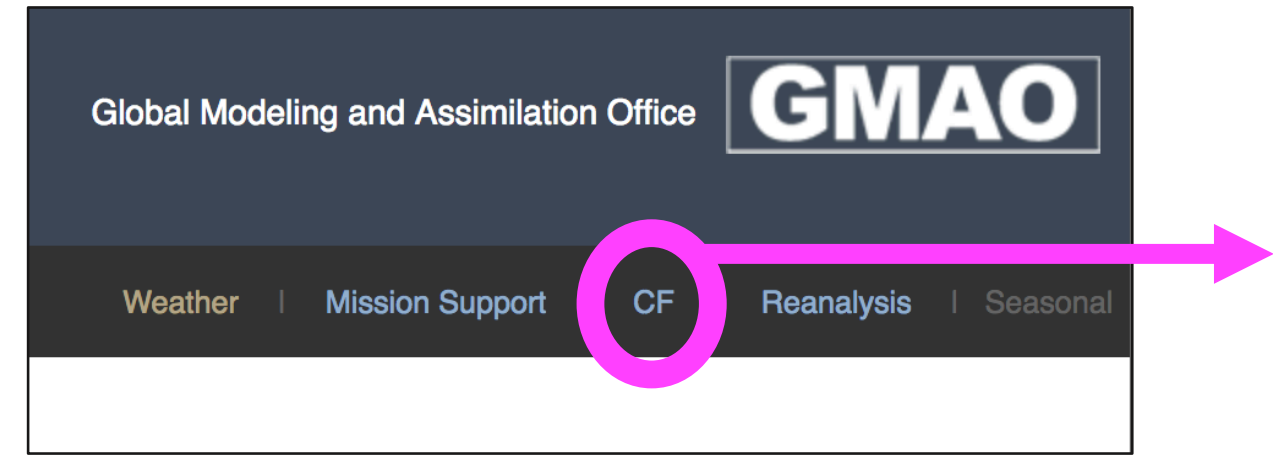

-

GMAO

Global Modeling and Assimilation Office 
FIELDS

$\mathrm{COSfc}$

Composition Forecast Maps

O3 Sfc PM2.5 Sfc

$\mathrm{SO} 2 \mathrm{Sfc}$

\section{REGIONS}

North America

\section{FORECAST INITIAL TIME}

$\triangle$

11Jun2019 12z

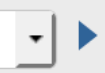

FORECAST LEAD HOUR

4 054h 13Jun2019 18z I

\section{Model forecast $\mathrm{O}_{3}$}

https:/fluid.nccs.nasa.gov/cf
NASA

GMAO

NASA/GMAO - GEOS CF Forecast Initialized on 12z 06/11/2019 Surface $\mathrm{O}_{3}$

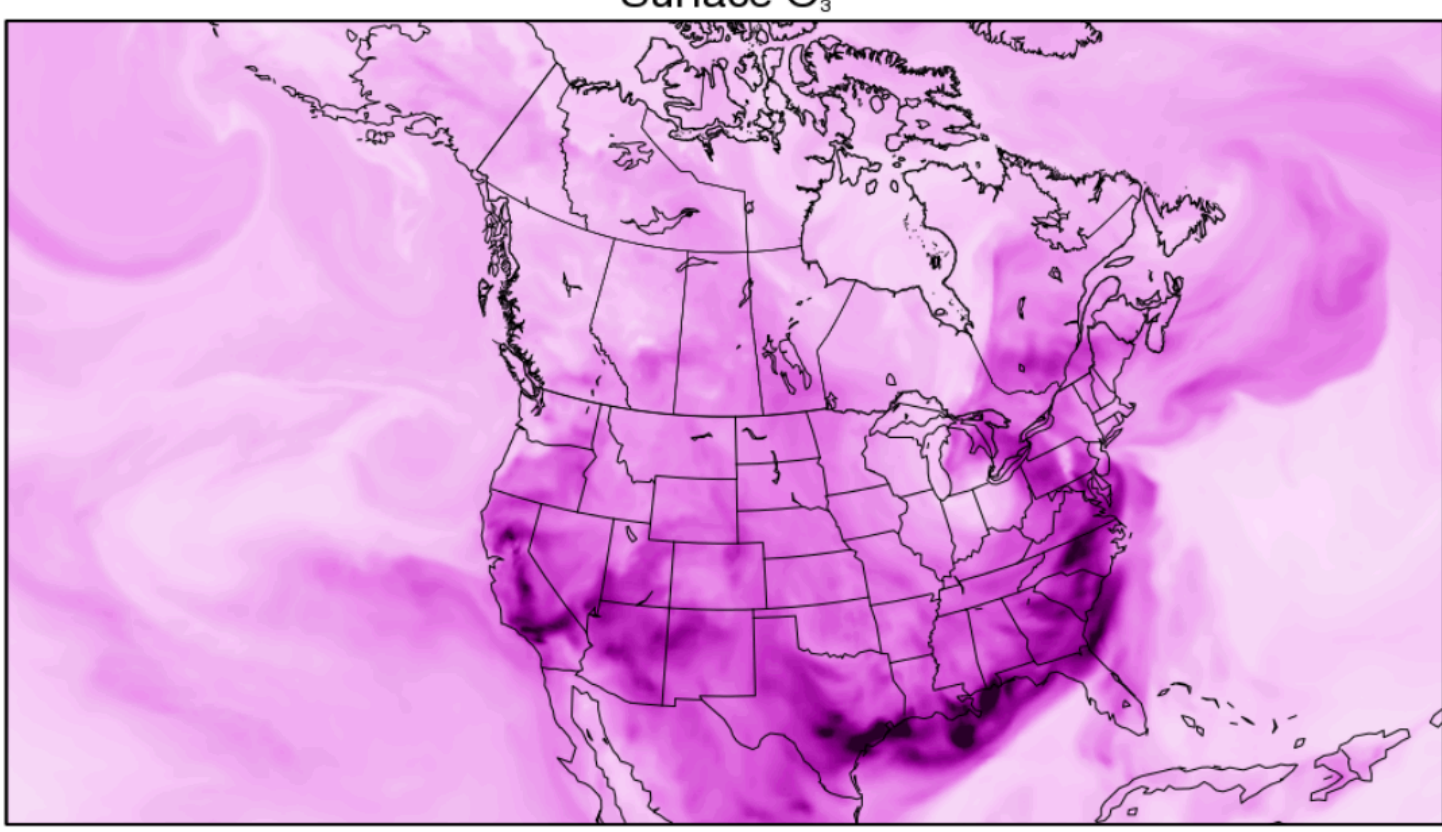

$054 \mathrm{hr}$ forecast valid Thu $18 \mathrm{z}$ 2019-06-13

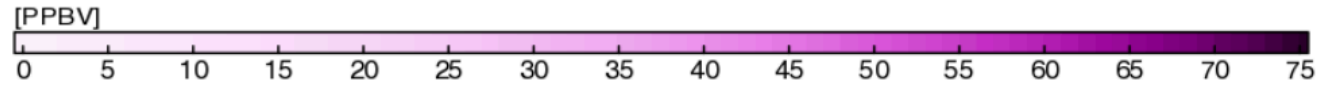




\begin{tabular}{|l|}
\hline \multicolumn{1}{|c|}{ NATIONAL } \\
\hline Raleigh \\
\hline WORLD \\
\hline Select a Station \\
AERONET \\
\hline Select a Station \\
\hline
\end{tabular}

MEGACITIES

Select a Station

ACTIVE CAMPAIGNS Select a Station

$$
\begin{array}{lll|l|l|l|}
\hline \mathrm{CO} & \mathrm{NO}_{2} & \mathrm{O}_{3} & \mathrm{PM} 2.5 & \mathrm{SO}_{2} \\
\hline
\end{array}
$$

$\mathrm{O}_{3}$ at Raleigh
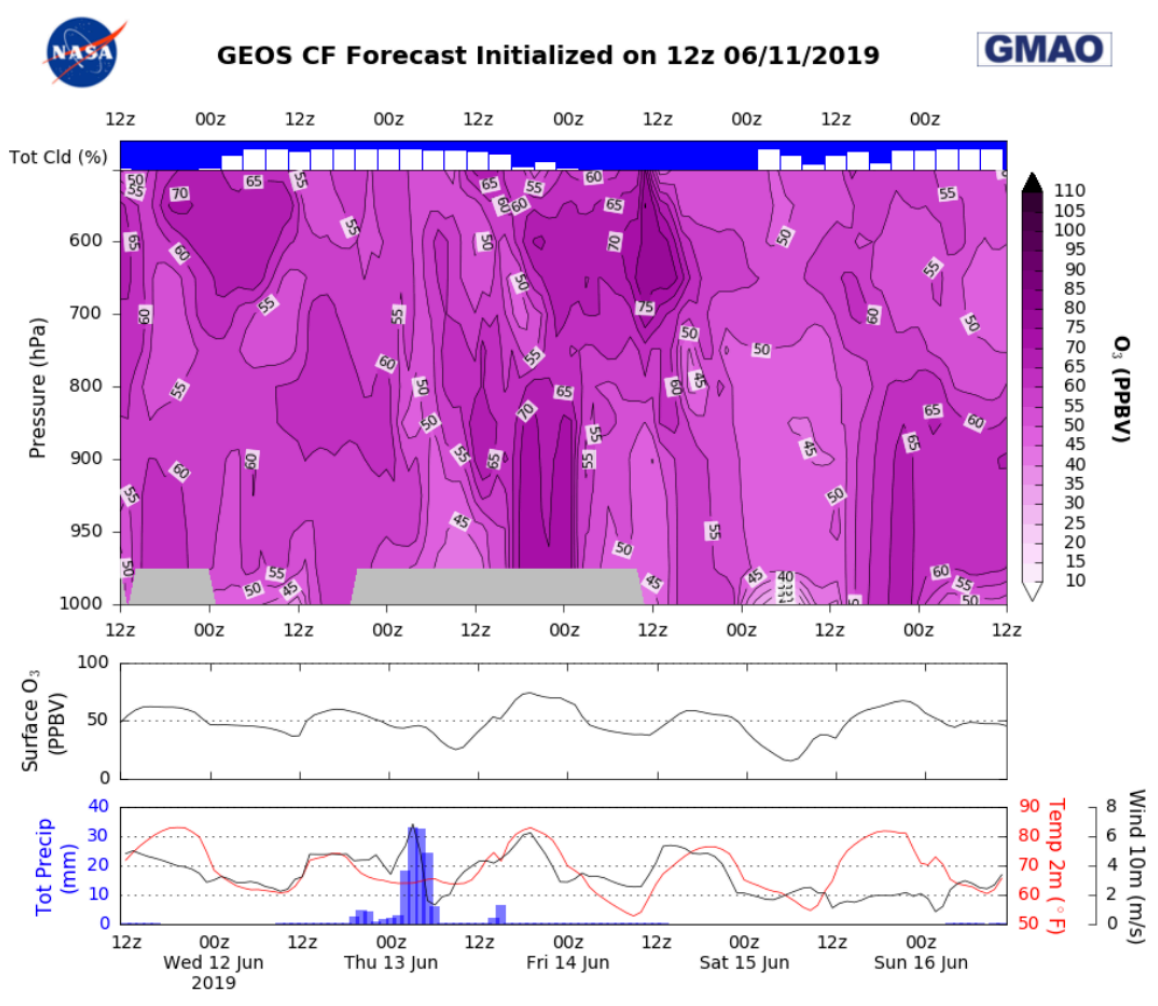

Surface $\mathrm{O}_{3}$

Meteorology 


\section{Summary}

- GEOS-CF produces daily global air quality forecasts at $25 \mathrm{~km}$ (16 miles) horizontal resolution

- Output available at fluid.nccs.nasa.gov/cf Under development:

- Assimilation system for trace gases $\left(\mathrm{O}_{3}, \mathrm{NO}_{\mathrm{x}}, \mathrm{CO}, \&\right.$ others $)$
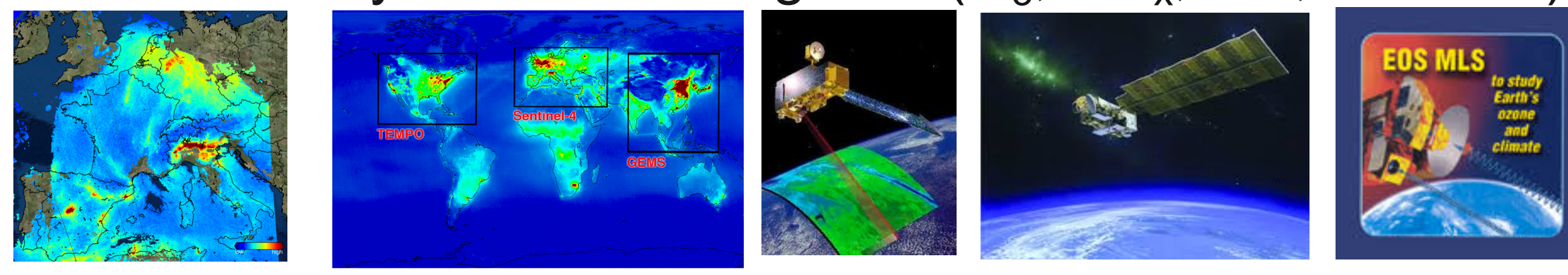

k.e.knowland@nasa.gov 
National Aeronautics and Space Administration

\section{4. unversirir of york

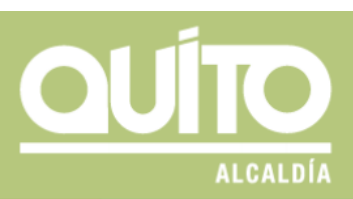

NYU Airlabs
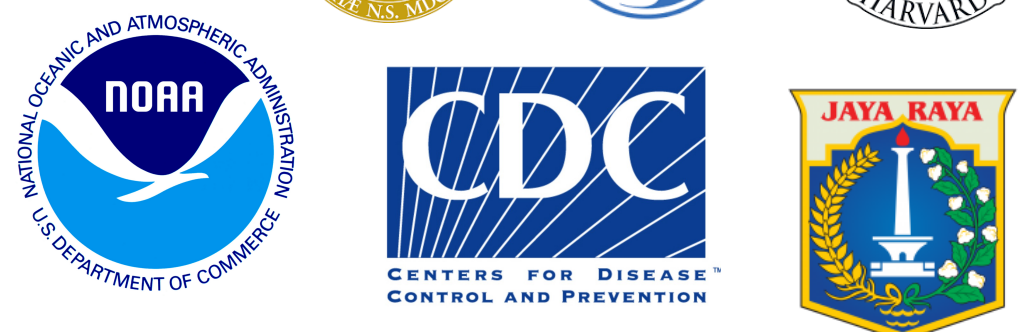

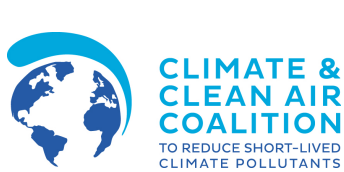

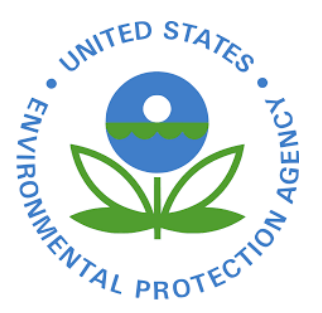

$\checkmark$ HawaDawa
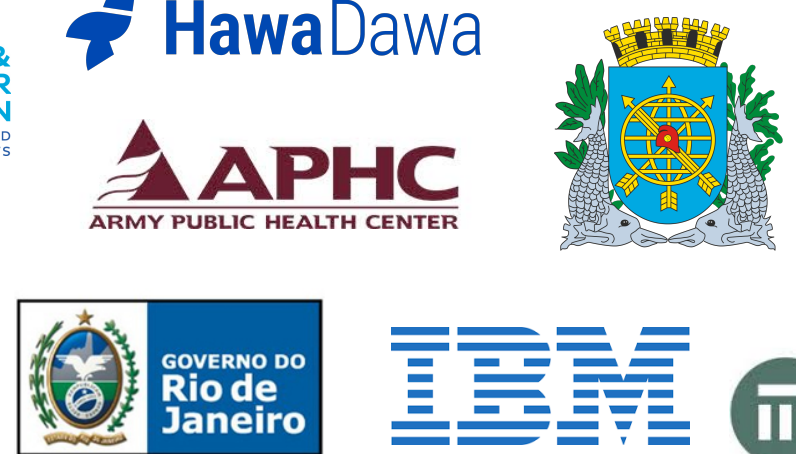

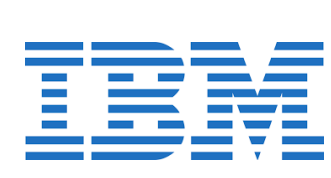

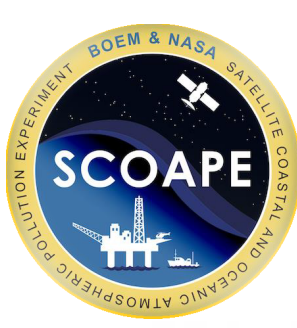

III)
Swiss Re

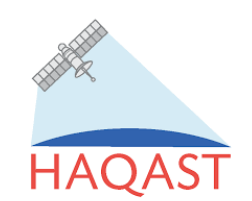

developmentseED
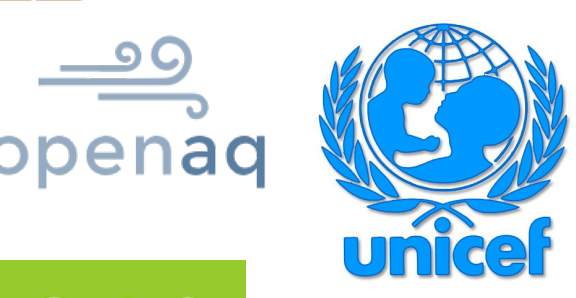

C40

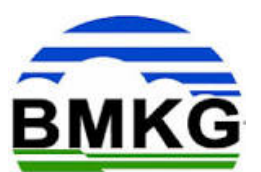
CITIES

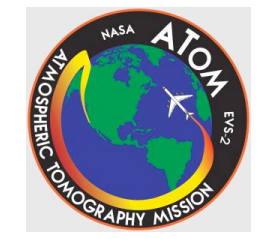




\section{7-10-01 00:30 UTC}

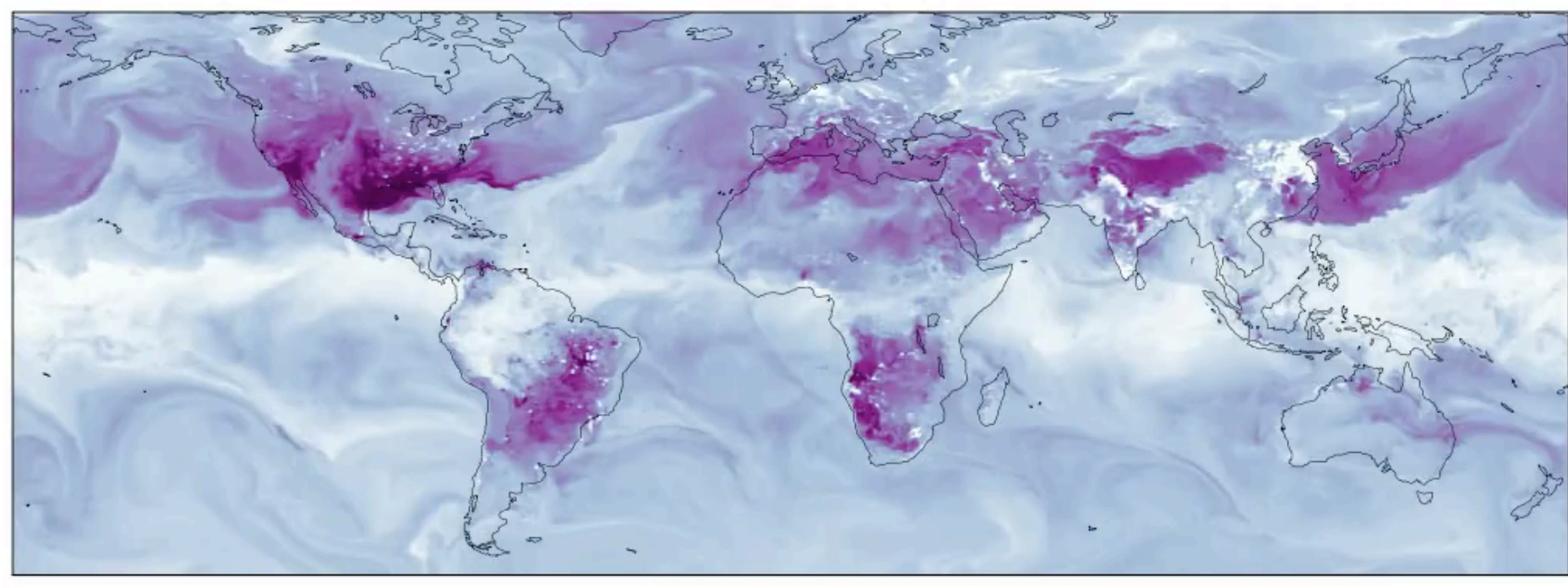

10

20

30

40

50

60

Surface ozone [ppbv]

https:/fluid.nccs.nasa.gov/cf 